EPJ Web of Conferences 16, 07002 (2011)

DOI: $10.1051 /$ epjconf/20111607002

(C) Owned by the authors, published by EDP Sciences, 2011

\title{
Spectral and polarimetric characterization of gazeous and telluric planets with SEE COAST
}

\author{
A. Boccaletti ${ }^{1, a}$, P. Baudoz ${ }^{1}$, D. Mawet ${ }^{2}$, J. Schneider $^{1}$, G. Tinetti ${ }^{3}$, \\ R. Galicher ${ }^{1}$, D. Stam ${ }^{4}$, C. Cavarroc ${ }^{5}$, J. Hough ${ }^{6}$, P. Doel ${ }^{3}$, D. Pinfield ${ }^{6}$, \\ C.-U. Keller ${ }^{7}$, J.-L. Beuzit ${ }^{8}$, S. Udry ${ }^{9}$, A. Ferrari ${ }^{10}$, E. Martin ${ }^{11}$, F. Ménard ${ }^{9}$ \\ and E. Sein ${ }^{12}$
}

${ }^{1}$ Observatoire de Paris, Meudon, France

2 JPL/CalTech, Pasadena, CA, USA

${ }^{3}$ Department of Physics and Astronomy, UCL, London, UK

${ }^{4}$ SRON, Utrecht, The Netherlands

${ }^{5}$ CEA/SAp, Saclay, France

${ }^{6}$ Centre for Astrophysics Research, University of Hertfordshire, Hatfield, UK

${ }^{7}$ Utrecht University, The Netherlands

${ }^{8}$ Laboratoire d'Astrophysique de Grenoble, France

${ }^{9}$ Observatoire de Genève, Switzerland

${ }^{10}$ Laboratoire d'Astrophysique de Marseille, Marseille, France

${ }^{11}$ Centro de Astrobiología, Madrid, Spain

${ }^{12}$ Astrium, France

\begin{abstract}
SEE COAST stands for Super Earth Explorer - Coronagraphic Off-Axis Space Telescope. The concept was initially proposed to ESA for Cosmic Vision. None of the direct detection exoplanet proposals were selected in 2007 and we are now pursuing our efforts to consolidate the astrophysical program and the technical developments for the next call for proposal. The prime objective of SEE COAST is to contribute to the understanding of the formation and evolution of planetary systems. Exploring the diversity of these objects is therefore the main driver to define the instrumentation. In the next decade the improvement of radial velocity instruments and obviously temporal coverage will provide us with a large numbers of long period giants as well as telluric planets, namely Super Earths. Obtaining the spectral and polarimetric signatures of these objects in the visible range to measure atmospheric parameters (molecular composition, clouds, soils, ...) will be unique and with important scientific returns. A space mission complementary to near IR instruments like SPHERE, GPI, JWST and later ELTs for the full characterization of giants and Super Earths is a first secure step towards the longer term goal that is the characterization of telluric planets with mass and atmosphere comparable to that of the Earth. An overview of the astrophysical motivation and the trade-off that lead to a simple integrated concept of a space-based high contrast imaging instrument are given here.
\end{abstract}

\section{CONTEXT}

Radial Velocity technique has been one of the most prolific method to identify extrasolar planets in the past decade. The 400 objects detected so far (http://exoplanet.eu) have their mass and orbital parameters constrained and for transiting planets, the radius has been also measured leading to an estimation of

\footnotetext{
ae-mail: anthony.boccaletti@obspm.fr
}

This is an Open Access article distributed under the terms of the Creative Commons Attribution-Noncommercial License 3.0, which permits unrestricted use, distribution, and reproduction in any noncommercial medium, provided the original work is properly cited. 
the density ([17]). The comparison with our own Solar System already suggests a large diversity (in mass, eccentricity, semi-major axis ...). One of the next step is the study of exoplanet atmospheres and we must be also prepared for diversity. In that context, direct detection is required as we need to collect the planetary photons to perform for instance spectroscopy. From space, transit spectroscopy of unresolved systems has been able to identify molecular species in the near and mid IR ([26, 28]). But more interestingly in our context, a few self-luminous planets have been already detected either from the ground and space using direct imaging $([13,16,19])$ and temperature and mass have been estimated from evolutionary models. In addition, direct imaging covers a parameter space that is complementary to Radial Velocity and transit as it is more sensitive to long period. Since the first detection of an exoplanet back in 1995, several projects of direct imaging have been developed (a review of projects is given in [6]) The first generation of instruments tuned for exoplanet observations will be on-sky by 20112012 at VLT ([7]), Gemini ([18]) and Subaru ([27]) but will mostly be sensitive to young and massive planets that are self luminous as contrast will be limited to $10^{-7}$ in the near IR. JWST will also open an interesting area as it is rather optimized for mid IR observations and that similar contrast will correspond to cooler planets than those accessible with planet finders on the ground $([5,8,15])$. It is anticipated that Extremely Large Telescopes (ELTs) on the ground will be able to access marginally to the realm of telluric planets and that large-scale space missions will be further required. Among telluric planets, Super-Earths (telluric planets that are more massive than the Earth) are receiving now a lot of attention as they can be also habitable but would be brighter than Earths-analogs. This ever-growing interest for Super Earths has led us to propose a space telescope operated in the visible for spectroscopic and polarimetric analysis of these objects. SEE COAST (Super Earth Explorer - Coronagraphic Off-Axis Space Telescope) was first proposed to Cosmic Vision at ESA in 2007 but not selected. The science potential and requirements are briefly described along this paper.

\section{ASTROPHYSICAL DRIVERS}

The core science program of SEE-COAST is to explore the diversity of planets especially focusing on Super Earths and mature Jovian planets. This goal is intermediate (scientifically and timely) in between the science program of 8-m class ground-based planet finders and more ambitious space missions geared towards the remote detection of life on Earth analogs (whatever the technique considered). Therefore, a mission like SEE COAST could be operated in parallel to ELTs on the ground with the objective to contribute to the characterization of the lowest mass gaseous planets and the most massive telluric ones.

A deep characterization of a planet requires the knowledge of physical parameters such as the mass, the radius, the atmosphere composition, the presence of clouds, the surface properties, and possibly the presence of biosignatures. Setting such an ambitious goal requires several tools to measure the planet signals. SEE COAST proposes to implement angularly resolved spectroscopy and polarimetry. A important aspect is the need for several visits as the planet characteristics are expected to vary in time (this is unique to space-based telescopes). Spectroscopy provides very rich information, such as the species present in the atmosphere, planetÕs cloud coverage and, if applicable, its surface properties. In addition, the polarimetric signal of jovian and telluric planets depends on wavelength and shows strong complementarities with spectroscopy to characterize planetary surfaces and clouds ([24]). As this program is certainly time consuming, the main observing strategy will be pointing to targets instead of surveys as we anticipated that in the coming years Radial Velocity instruments will provided many targets accessible with SEE-COAST. Survey modes might be restricted to specific targets.

The SEE-COAST program focuses especially on the following physical parameters :

- Mass: The mass is derived from RV measurements coupled with several observations along the orbit to constraint the inclination angle of the planet orbit.

- Radius: Unless transit measurements are available, which is unlikely for direct imaging targets, the estimation has to rely on atmospheric models. It is therefore essential to secure these models on transiting planets. 
Table 1. Summary of instrumental requirements.

\begin{tabular}{ll}
\hline Parameter & Value \\
\hline telescope diameter & $1.5-1.8 \mathrm{~m}$ \\
spectral range $(2$ channels $)$ & $0.4-1.25 \mu \mathrm{m}$ \\
spectral resolution & $40-80$ \\
min. field of view & $3^{\prime \prime}$ \\
wide field capability & $30^{\prime \prime}$ \\
optical quality & $\lambda / 100 \mathrm{rms}$ \\
contrast & $10^{-9}-10^{-10}$ (goal) \\
inner working angle & $2($ goal) $-3 \lambda / \mathrm{D}$ \\
polarimetric accuracy & $10 \%$ \\
nulber of visits & $>3-5$ (goal) \\
\hline
\end{tabular}

- Atmosphere: This is obviously the main outcome of spectral characterization (either based on total flux or polarized light measurements). Visible and near Infrared spectrum is rich of spectral features like $\mathrm{H}_{2} \mathrm{O}, \mathrm{O}_{2}, \mathrm{CH}_{4}, \mathrm{CO}_{2}, \mathrm{NH}_{3}$ and they are large enough to be detected with spectral resolution of 40 to 80 . Some of these molecules have been already detected in transiting hot giant planets in the IR $([4,25,26,28])$. There is no doubt that 8 -m class planet finders, JWST and SPICA will obtain spectra or colors of some warm giants in the near IR and possibly some mature also in the mid IR. However, tracing the same molecules in the visible is very important to better constrain atmospheric parameters (temperature and pressure profiles, albedo) and to secure detection. As for Super Earths, the chemically reactive molecules $\mathrm{H}_{2} \mathrm{O}, \mathrm{O}_{2}$ or $\mathrm{CO}_{2}$ will be search for as they are considered as biosignatures. Water is a necessary solvent for life to emerge. It is probably common and already found in a gas giant outside the solar system ([28]). Carbon dioxide would indicate that carbon is available for the biosphere and that greenhouse effect may play a important role. Finally, oxygen is so reactive that it must be produced at enormous rate to persist and is obviously associated to the presence of life. Moreover it is unique to the visible range and then future programs of life remote detection will necessarily have to combine multi-wavelength informations from the visible to the mid-IR.

- Surface: Oceans and continents have a different temperatures and albedos and bidirectional reßection functions. They typically present a variation of a factor $\sim 5$ in reßected light. The degree of polarization also strongly depends on the surface nature and is complementary to spectroscopy (Figure ???). In addition, the planetary rotation will modulated this signal and seasonal variations are also expected. So in principle the duration of the day can be derived with consequence on dynamics. However, the detectability of such physical parameters is certainly a difficult task as integration time might be several hours or days. Modeling will be required to break degeneracies and try to recover informations on the planetary surface and rotation. Several visits are definitely required.

More details about the science program of SEE-COAST are given in [23].

\section{INSTRUMENTAL REQUIREMENTS}

The ambitious program described above obviously requires high angular resolution, high contrast capabilities, spectral coverage, adequate spectral resolution and polarimetric accuracy. A small $1.5 \mathrm{~m}$ telescope in space if equipped with high contrast imaging facilities and appropriate instruments can carry out this program for nearby stars $(<20 \mathrm{pc})$ and will nicely complement 8 -m class planet finders and JWST and will prepare for more ambitious programs that aim to detect Earth analogs and search for traces of life. High-level mission requirements are listed in Table 1. 

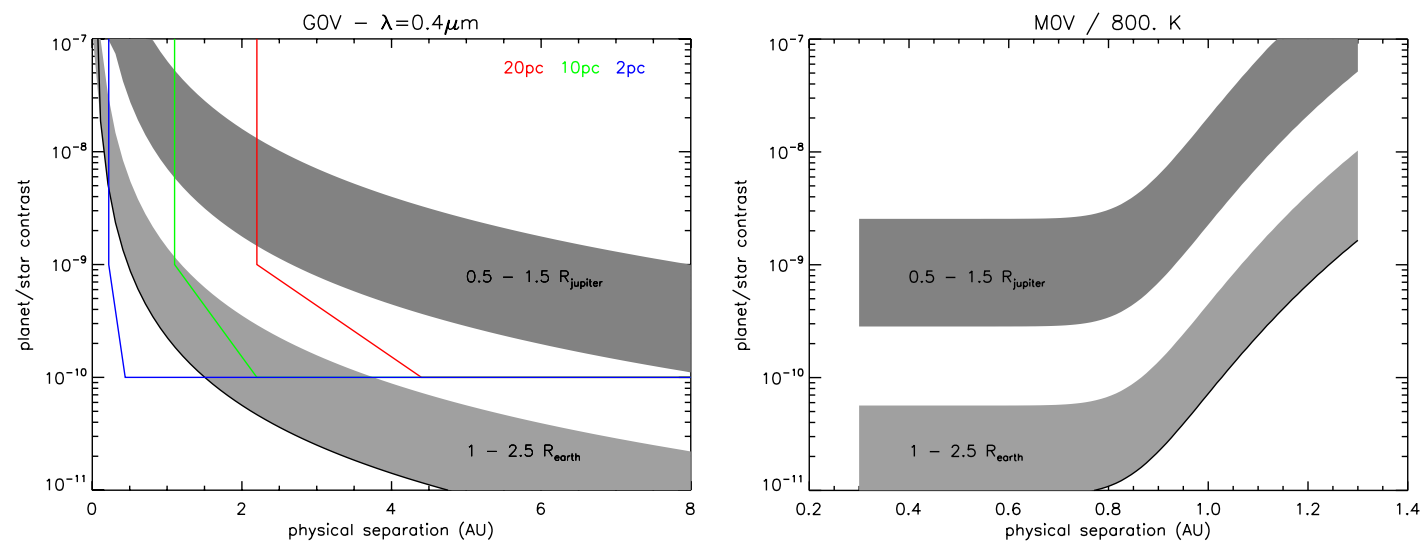

Figure 1. Left plot shows the star to planet contrast compared to instrumental specifications (colored curves) scaled according to wavelength and star distance (for an albedo of 0.5 , a G0V star and $\lambda=0.4 \mu \mathrm{m}$ ). At the end of the visible spectral range $(\lambda=0.8 \mu \mathrm{m})$ the instrumental contrasts are twice further away from the star. Right plot shows the contrast against wavelength for a self luminous warm planet $(800 \mathrm{~K})$ at $5 \mathrm{AU}$ from a M0V star.

The focal instrument should provide spectroscopy as well as polarimetric measurements in a narrow field of view $\left(3 \times 3^{\prime \prime}\right)$ between 0.4 and $1.25 \mu \mathrm{m}$. The optical implementation of such a concept deserve a thorough study but Integral Field Spectrograph are promising and have been already tailored for exopanet detection ([1]). For that, the heritage of SPHERE ([7]) and EPICS ([14]) will be a major advantage for the technical aspects and instrumental modeling.

To define the parameter space of the detectable planets we compared the theoretical contrasts in reflected light for 2 classes of planets, the giants and tellurics, compared to instrumental specifications. Figure 1 shows star/planet contrasts for reflected light and in the favorable case of warm self-luminous planets. Telluric planets are accessible for very nearby stars within 5 AUs providing small inner working angle $(2 \lambda / \mathrm{D})$ and large contrast $\left(10^{-10}\right)$ are met. Even an Earth like planet can be detected if any around the closest star. Spectroscopy will be feasible on giant planets within 10 AUs for stars closer than $40 \mathrm{pc}$. The shortest wavelengths $(0.4 \mu \mathrm{m})$ are clearly more favorable for detection (contrast curves scale linearly with $\lambda$ ). Young planets are also preferential targets for SEE COAST. At large temperatures $(>600-800 \mathrm{~K})$ the thermal emission produces a significant signal at $1.2 \mu \mathrm{m}$ and translates into contrasts of about $10^{-9}$ for tellurics and $10^{-7}$ for giants around $\mathrm{M}$ stars (independently of separation). Hence, spectro-polarimetry of young objects is feasible around distant stars (50-100 pc).

\section{MISSION CONCEPT}

SEE-COAST is designed to directly detect exoplanets against the stellar diffracted light. The main critical aspects to reduce this background noise are: 1/ the correction of wavefront errors (WFE), $2 /$ the suppression of the starlight with a coronagraph and 3/ the calibration of residuals not corrected by the 2 previous stages. Several studies on small space telescopes for exoplanet imaging $([12,30])$ are ongoing and then many instrumental solutions are being investigated. Therefore, among the solutions that we consider relevant for SEE-COAST we only focused on a few, briefly described below.

WFE requirements can be obtained with good optical quality at the primary mirror but the implementation of a Deformable Mirror (DM) may be mandatory. The implementation of a DM in a space telescope has already been studied in the context of TPF-C ([29]) and SPICA ([9]). It is admitted that the optimal place to perform wavefront sensing is at the detector plane. Several algorithms have been developed in that context. In our group we are studying the concept of the Self Coherent Camera (SCC, [2]) which is able to measure the wavefront errors with a high precision and nicely fits in a 
coronagraph design. The analytical and numerical study has been carried out ([10]) and now a testbed is being implemented.

The coronagraphic device should provide high contrast, high throughput, and low chromaticity. In that context, several concepts are being prototyped for space telescopes like the PIAA ([11]). We are pursuing two concepts based on phase masks to improve the achromatic behavior. First, the optical vortex based on the initial idea of the Annular Groove Phase Mask ([20]). A technique using Liquid Crystal Polymer has now made possible the manufacturing of such masks which can be made achromatic ([21]) and recent results have shown contrast of $10^{-7}$ ([22]). Optical Vortices can be made less sensitive to stellar leakage at the expense of a larger inner working angle. The second solution is called the multistage 4 quadrant phase mask that has already demonstrated very high contrast of the order of $10^{-9}$ in the lab at a few $\lambda / D([3])$.

Finally, after this two stages it is still possible to further calibrate a posteriori the residuals using a diversity approach. For instance, the need for a full spectral coverage will make necessary the use of an Integral Field Spectrograph. As planetary and stellar spectra are different, planet light and stellar speckle can be disentangle providing chromaticity is small. This will require an optimization of the optical system but we note that a solution has been already proposed ([1]) and implemented for SPHERE the VLT planet finder and is now considered for EPICS on the european ELT. Similarly, the SCC is able to discriminate between photons from the star and from the planet and can provide, in addition to wavefront sensing, another tool to reduce the speckle background.

\section{CONCLUSION}

The current knowledge on extrasolar planets justifies an intermediate imaging mission to fill the gap between planet finders on 8-m class (mostly focused on warm giants) and more ambitious flagship missions (optimized for telluric planets). SEE COAST falls in this slot as other small telescopes like PECO and ACCESS on the US side $([12,30])$ an could eventually be merged. Technology has now a lot progressed through lab experiments in both coronagraphy and wavefront control to certify that even a small telescope if properly optimized can achieve unprecedented contrast, surpassing the performance of ground-based 8-m class telescopes and also JWST, in what concern direct imaging of extrasolar planets. Therefore, a coronagraphic mission like SEE-COAST is an interesting precursor mission to carry out the physico-chemical characterization of giant planets and some super-Earths before more ambitious missions like large coronagraphic (4-8 m class) missions, large interferometers, or external occulters.

SEE-COAST was initially proposed in 2007 for ESA Cosmic Vision call for missions. Actually, none of the missions designed for direct imaging of exoplanets was selected on the basis of cost issues, non mature technology and restricted science program (in regards of the cost investment). A new call for mission will be presumably released in late 2010. There are several areas in which we are improving our proposal. On the astrophysical aspects, a new analysis of performance and statistics of detection is being conducted in the detail, and other observing modes will be considered (transit photometry, and astrometry). As for technology, many concepts are developed around the world and we are paying attention to a few of them as described above. In addition, a global system analysis will be conducted in partnership with ASTRIUM France.

\section{References}

[1] Antichi, J., Dohlen, K., Gratton, R., et al., ApJ 695, 1042 (2009)

[2] Baudoz, P., Boccaletti, A., Baudrand, J., Rouan, D., IAU Colloq. 200, 553 (2006)

[3] Baudoz, P., Galicher, R., Baudrand, J., Boccaletti, A., SPIE Conf. Serie 7015, 70156 (2008)

[4] Beaulieu, J.P., Kipping, D.M., Batista, V., et al., arXiv 0909.0185 (2009)

[5] Boccaletti, A., Baudoz, P., Baudrand, J., et al. , Advances in Space Research 36, 1099 (2005) 
[6] Boccaletti, A., arXiv 0910.4339 (2009)

[7] Beuzit, J.-L., Feldt, M., Dohlen, K., et al., The Messenger 125, 29 (2006)

[8] Doyon, R., Rowlands, N., Hutchings, J., et al., SPIE Conf. Serie 7010 (2008)

[9] Enya, K., Abe, L., Haze, K., et al., SPIE Conf. Serie 7010, 70102 (2008)

[10] Galicher, R., Baudoz, P., Rousset, G., A\&A 488, 9 (2008)

[11] Guyon, O., Pluzhnik, E., Galicher, R., et al., ApJ 622, 774 (2005)

[12] Guyon, O., Angel, J.-R.-P., Backman, D., et al., SPIE Conf. Serie 7010, 59 (2008)

[13] Kalas, P., Graham, J., Chiang, E., et al., Science 322, 1345 (2008)

[14] Kasper, M.-E., Beuzit, J.-L., Verinaud, C., et al. , SPIE Conf. Serie 7015, 46 (2008)

[15] Krist, J., SPIE Conf. Serie 6693 (2007)

[16] Lagrange, A.-M., Gratadour, D., Chauvin, G., et al., A\&A 493, L21 (2009)

[17] Léger, A., Rouan, D., Schneider, J., et al., arXiv 0908.0241 (2009)

[18] Macintosh, B., Graham, J., Palmer, D., et al., SPIE Conf. Serie 6672 (2006)

[19] Marois, C., Macintosh, B., Barman, T., et al., Science 322, 1348 (2008)

[20] Mawet, D., Riaud, P., Absil, O. and Surdej, J., ApJ 633, 1191 (2005)

[21] Mawet, D., Serabyn, E., Liewer, K., et al., Optics Express 17, 1902 (2009)

[22] Mawet, D., Trauger, J., Serabyn, E., et al., SPIE Conf. Serie 7440 (2009)

[23] Schneider, J., Boccaletti, A., Mawet, D., Baudoz, P., et al., Exp. Astron. 23, 357 (2009)

[24] Stam, D., A\&A 482, 989 (2008)

[25] Swain, M., Vasisht, G., Tinetti, G., Nature 452, 329 (2008)

[26] Swain, M., Vasisht, G., Tinetti, G., et al., ApJ 690, 114 (2009)

[27] Tamura, M. and Hodapp, K. and Takami, H., et al., SPIE Conf. Serie 6269 (2006)

[28] Tinetti, G., Liang, M.-C., Vidal-Madjar, A., et al., ApJ 654, L99 (2007)

[29] Trauger, J. \& Traub, W., Nature 446, 771 (2007)

[30] Trauger, J., Stapelfeldt, K., Traub, W., et al., SPIE Conf. Serie 7010, 69 (2008) 